\title{
ON THE HAUSDORFF DIMENSION OF SOME SETS IN EUCLIDEAN SPACE
}

\author{
PAUL ERDÖS
}

Let $E$ be a closed set in $n$-dimensional space, $x$ a point not in $E$. Denote by $S(x)$ the largest sphere of center $x$ which does not contain any point of $E$ in its interior. Put $\phi(x)=E \cap \bar{S}(x)$. ( $\bar{A}$ denotes the closure of $A$.) Denote by $M_{k}$ the set of points for which $\phi(x)$ contains $k$ or more linearly independent points (that is, points which do not lie in any $(k-2)$-dimensional hyperplane). $M_{k}$ is defined for $k \leqq n+1$. In a previous paper I proved that $M_{2}$ has $n$-dimensional measure 0 and conjectured that $M_{k}$ has Hausdorff dimension not greater than $n+1-k$. In the present note we shall prove this conjecture. In my previous paper I also proved that $M_{n+1}$ is countable, but the proof there given applied only for the case $n=2$; now we are going to give a general proof.

Let $R$ be any set in $n$-dimensional space. Let $x \in R$. We define the contingent ${ }^{1}$ of $R$ at $x$ ( $\operatorname{contg}_{R} x$ ) as follows: The contingent will be a subset of the unit sphere. A point $z$ of the unit sphere belongs to $\operatorname{contg}_{R} x$ if and only if there exists a sequence of points $y_{1}, y_{2}, \ldots$ in $R$ converging to $x$ so that the direction of the vector connecting $x$ with $y_{j}$ tends to the direction of the vector connecting the center of the unit sphere with $z$. First we state the following lemma.

Lemma. Let there be given a set $R$ in n-dimensional space. Assume that for every $x, \operatorname{contg}_{R} x$ does not contain any point of the intersection of the unit sphere with a k-dimensional hyperplane going through its center (the hyperplane can depend on $x$ ). Then $R$ is contained in the sum of countably many surfaces of finite $(n-k)$-dimensional measure.

This lemma is well known. ${ }^{2}$

ThEOREM 1. Let $k<n+1$. Then $M_{k}$ is contained in the sum of countably many surfaces of finite $(n+1-k)$-dimensional measure. If $k=n+1$, then $M_{k}$ is countable. ${ }^{3}$

Received by the editors September 24, 1945.

1 G. Bouligand, Introduction d la geometrie infinitesimale directe. Also Saks, Theory of the integral.

2 Saks, ibid. pp. 264-266 and pp. 304-307. Also Roger, C. R. Acad. Sci. Paris vol. 201 (1935) pp. 871-873. 1939.

For $n=2$ this theorem is proved by C. Pauc, Revue Scientifique, August, 
Remark. This clearly means that the Hausdorff dimension of $M_{h}$ $(k \leqq n+1)$ is not greater than $n+1-k$.

Let us first consider the case $k=n+1$. Assume that $x \in M_{n+1}$. Let $z_{i} \in \phi(x), i=1,2, \cdots, n+1$, and assume that the $z$ 's are linearly independent. Denote by $f(x)$ the maximum value of the volume of the simplices determined by the $z$ 's (since $\phi(x)$ is closed the maximum is attained). Define now $N_{n+1}{ }^{(c)}=N$ to consist of all the points $x \in M_{n+1}$ for which $f(x) \geqq c$. It clearly will be sufficient to show that $N$ is countable (for every $c$ ). In fact we shall show that $N$ is isolated (in other words no $x \in N$ is a limit point of $N-x$ ), that is, we shall prove that for every $x \in N \operatorname{contg}_{N} x$ is empty. If this would not hold then $N$ would contain an infinite sequence of points $y_{j}$ coverging to $x$ so that the direction of the line connecting $x$ with $y_{j}$ would converge to a fixed direction. Let $Z_{j}$ be a point of $\phi(x)$ which is closest to $y_{2}$, and let $A_{i}$ be the (unique) hyperplane through $Z_{j}$ perpendicular to the segment $x y_{j}$. It is easy to see that as $j \rightarrow \infty, A_{j}$ converges to a limiting hyperplane $A$. Moreover it is easily seen that the set $\phi\left(y_{j}\right)$ is ultimately contained in any preassigned neighborhood of $A$. Thus for large enough $j$, the volume $f\left(y_{j}\right)$ must be less than $c$, an evident contradiction; this completes our proof.

Next we prove our theorem in the general case. Let $k \leqq n$ and define $M_{k}^{\prime}$ to be the set of all points $x$ for which the maximum number of linearly independent points in $\phi(x)$ is exactly $k$. It will clearly be sufficient to show that $M_{k}^{\prime}$ is contained in the sum of countably many surfaces of finite $(n+1-k)$-dimensional measure. Let $x \in M_{k}^{\prime}$, and let $f(x)$ be the maximum volume of the $k$-dimensional simplices formed from the points $z_{i}, i \leqq k+1$, where $z_{i} \in \phi(x) . x \in M_{k}{ }^{\prime(0)}=N^{\prime}$ if $f(x) \geqq c$. Let $x \in N^{\prime}$, and $z_{i}, i \leqq k+1$, be the points which determine a simplex of maximal volume. Then a simple geometrical argument (similar to the previous one) shows that contg $N^{\prime} x$ consists only of the directions through $x$ which are perpendicular to the hyperplane determined by the $z_{i}$ 's, $i \leqq k+1$. Thus our theorem follows from the lemma.

Let $E$ be a closed set, $x \notin E$. Denote by $g(x)$ the distance of $x$ from $E$. It has been proved that $g(x)$ has a derivative $-\cos \alpha$ in every direction $(x, y)$, where $\alpha$ is the smallest angle formed by the direction $(x, y)$ with the direction $(x, z), z$ in $\phi(x)$. Clearly if $x \in E$ the derivative of $g(x)$ can be 0 . We shall show that the derivative of $g(x)$ is 0 for almost all points of $E$.

${ }^{4}$ Mises, C. R. Acad. Sci. Paris vol. 205 (1937) pp. 1353-1355. See also Golab, ibid. vol. 206 (1938) pp. 406-408 and Bouligand, ibid. vol. 206 (1938) pp. 552554. 
Let $x \in E$. Denote by $S(x, \epsilon)$ the sphere of center $x$ and radius $\epsilon$. Denote by $G(x, \epsilon)$ the greatest distance of the points of $\bar{S}(x, \epsilon)$ from $E$. We are going to prove the following theorem.

Theorem 2. For almost all points of $E$ (that is, for all points of $E$ except a set of $n$-dimensional measure 0 )

$$
\lim G(x, \epsilon) / \epsilon=0 .
$$

It is well known that almost all points of $E$ are points of Lebesgue density 1 . Let $x$ be such a point, and suppose that

$$
\lim G(x, \epsilon) / \epsilon \neq 0 .
$$

This means that there exists an infinite sequence $\epsilon_{i}$ and points $z_{i}$, $z_{i} \in \bar{S}\left(x, \epsilon_{i}\right), \epsilon_{i} \rightarrow 0$, such that the distance of $z_{i}$ from $E$ is greater than $c \epsilon_{i}$, where $c>0$. But this clearly means that $x$ can not have Lebesgue density 1 . This contradiction establishes our theorem.

UNIVERSITY OF MICHIGAN

\section{ON MONOTONE RETRACTABILITY INTO SIMPLE ARCS}

\section{G. T. WHYBURN}

In recent work on the area of surfaces Rado [1] has had occasion to use the following properties as applied to locally connected continua $A$ :

( $\pi$ ) Every simple arc in $A$ is a monotone retract of $A$;

(II) Every monotone image of $A$ has property ( $\pi$ ).

Radó has noted that (II) implies ( $\pi$ ) and that the sphere and 2-cell each have (II). In this paper it will be shown that (1) for locally connected continua in general, property (II) is equivalent to unicoherence, (2) for plane locally connected continua, property $(\pi)$ is equivalent to unicoherence, and (3) every closed 2-dimensional connected manifold has property $(\pi)$.

To clarify our meaning, we recall that a continuum is compact, connected and metric. A continuous mapping $f(A)=B$ on a continuum $A$ is monotone provided $f^{-1}(y)$ is a continuum for $y \in B$. If 1945.

Presented to the Society, September 17, 1945; received by the editors September 8,

${ }^{1}$ Numbers in brackets refer to the Bibliography at the end of the paper. 